Short communication

\title{
Detection of avian influenza virus subtype H5 using a biosensor based on imaging ellipsometry
}

\author{
Cai Qi ${ }^{a, c, e, 1}$, Xin-Sheng Tian ${ }^{b, c, 1}$, She Chen ${ }^{a, c}$, Jing-Hua Yan ${ }^{b}$, Zhen Cao ${ }^{d}$, Ke-Gong Tian ${ }^{d}$, \\ George F. Gao ${ }^{\mathrm{b}, *}$, Gang Jin ${ }^{\mathrm{a}, *}$ \\ a Institute of Mechanics, Chinese Academy of Sciences, Beijing 100190 China \\ b CAS Key Laboratory of Pathogenic Microbiology and Immunology, Institute of Microbiology, Chinese Academy of Sciences, Beijing 100101, China \\ ${ }^{\mathrm{c}}$ Graduate University, Chinese Academy of Sciences, Beijing 100049, China \\ d China Animal Disease Control Center, Beijing 100094, China \\ e Institute of Biophysics, Chinese Academy of Sciences, Beijing 100101, China
}

\section{A R T I C L E I N F O}

\section{Article history:}

Received 1 August 2009

Received in revised form 21 October 2009

Accepted 21 October 2009

Available online 30 October 2009

\section{Keywords:}

Avian influenza virus

Biosensor

Ellipsometry

Scanning near-field optical microscopy

\begin{abstract}
A B S T R A C T
A novel method is reported for the detection of avian influenza virus subtype $\mathrm{H} 5 \mathrm{using}$ a biosensor based on high spatial resolution imaging ellipsometry (IE). Monoclonal antibodies specific to $\mathrm{H} 5$ hemagglutinin protein were immobilized on silicon wafers and used to capture virus particles. Resultant changes on the surface of the wafers were visualized directly in gray-scale on an imaging ellipsometry image. This preliminary study has shown that the assay is rapid and specific for the identification of avian influenza virus subtype H5. Compared with lateral-flow immunoassays, this biosensor not only has better sensitivity, but can also simultaneously perform multiplexed tests. These results suggest that this biosensor might be a valuable diagnostic tool for avian influenza virus detection.
\end{abstract}

(C) 2009 Elsevier B.V. All rights reserved.

\section{Introduction}

According to September 2009 World Health Organization statistics, the number of cases of the avian influenza virus (AIV) H5N1 directly crossing barriers and infecting humans was 442, giving rise to 262 deaths (World Health Organization, 2009). In addition to H5N1, H9N2 and H7 subtypes have also been directly transmitted to humans (Malik Peiris, 2009). The current influenza pandemic has caused serious concern all over the world, and highlights the need for a highly sensitive, accurate and rapid diagnostic tool for routine laboratory tests and systematic surveillance programs.

Traditional methods for AIV detection and subtype identification are based on virus isolation (VI) in tissue culture or embryonating chicken eggs. Although VI is highly specific and sensitive, it is labor-intensive and takes 1-2 weeks to get results. While alternative methods such as nucleic acid sequence-based amplification (Lau et al., 2004), polymerase chain reaction (Payungporn et al., 2006), poly-crystalline silicon nanowire field-effect transistor (Lin et al., 2009), etc., testing of viral nucleic acids are highly sensitive and allow rapid diagnosis of influenza infection, they often

\footnotetext{
* Corresponding author. Tel./fax: +86 10 82544138; fax: +86 1064807598 .

E-mail addresses: gaof@im.ac.cn (G.F. Gao), gajin@imech.ac.cn (G. Jin).

${ }^{1}$ These authors contributed equally to this work.
}

require a relatively high level of sample manipulation that is inconvenient for infectious materials.

Compared with the above methods, the advantages of a biosensor based on imaging ellipsometry (BIE), such as high-throughput allowing for multiplexed analysis, and quantitative, label-free and rapid testing, are evident. While applications of BIE have mainly been focused on the biomedical fields (Qi et al., 2009a; Jin, 2008; Qi et al., 2006; Bae et al., 2004; Wang and Jin, 2003a,b) it is only recently that a basic model for virus detection using BIE has been established (Qi et al., 2009b). However, this method has not yet been applied for detection of infective viruses, particularly for the detection of whole virus particles causing human infectious diseases.

The purpose of this study was to specifically identify different AIV subtypes using a micro-array with multi-ligands. The key characteristic of our micro-array is that AIV antibody immobilization is oriented by means of an interaction between protein $A$ and the Fc fragment of the antibody. Each kind of antibody in the micro-array captures its corresponding whole avian influenza cystovirus, which is then quantified by the BIE.

\section{Experiment}

\subsection{BIE principle}

BIE has been developed in our laboratory for the purpose of performing parallel immunoassays and is based on a combination of an 

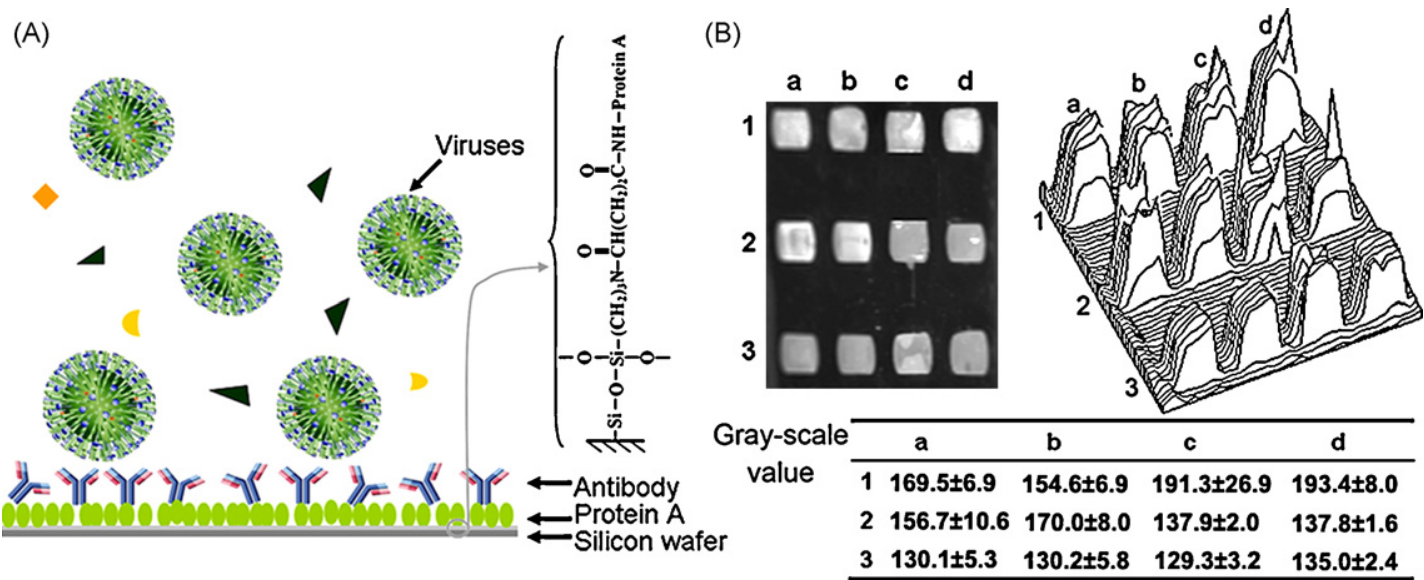

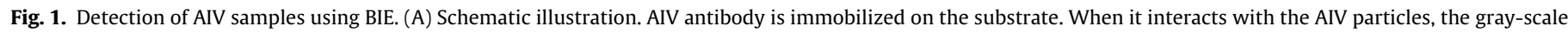

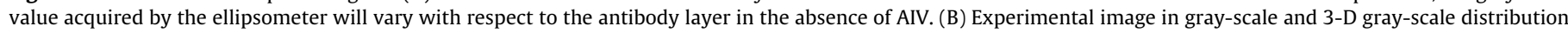

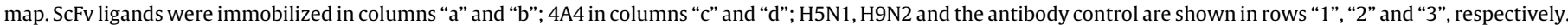

integrated microfluidic reactor array system and imaging ellipsometry (IE). The microfluidic system is used for surface patterning and array production, and for solution delivery, ligand immobilization and target capture. IE is used for reading the protein arrays.

The microfluidic system has four main parts: sample plate, multi-cell array, micro-channels and pumps. A multi-cell array is formed when a polydimethyl-siloxane (PDMS) pattern mould is in contact with the surface of a silicon substrate, each cell having an inlet and an outlet for solution passage (Wang et al., 2006). The inlet micro-channels are put into sample plate and outlet micro-channels are connected with pumps (ISM939, ISMATEC, Switzerland. www.ismatec.com) offering negative pressure. Different antibody ligands are delivered to different cells for immobilization using the microfluidic system so that the surface is patterned homogeneously and simultaneously in an array format. The system's simple channel junctions can be used in serial or parallel formats to analyze single or multiple samples simultaneously.

IE is a display technique for ultra-thin film and surface characterization (Jin et al., 1996). The incident wave of polarized light irradiates the sample as a probe beam and is thereby modified resulting in the reflective or transmission beam having the ability to carry sample information, such as protein layer thickness. When IE is used to detect layer thickness, the reflection intensity is represented in gray-scale, and the variation in layer thickness leads to changes in the gray-scale value. If the refractive index is invariant, the gray-scale value will be directly proportional to the thickness of the protein layer within the range of 0-30 nm layer thickness, i.e. $I=k d$, where $I$ is the light intensity and $d$ is the layer thickness (Chen et al., 2007). Under these conditions $k$ is a constant and can be determined from a protein layer with known grayscale values and known thickness (Arwin et al., 1993). At the same time there is a relationship between surface concentration and film thickness: surface concentration $\left(\mu \mathrm{g} / \mathrm{cm}^{2}\right) \approx K \times d$, where $K=0.12$ (Stenberg and Nygren, 1983). Thus the gray-scale value directly reflects layer thickness and surface concentration. The higher the gray-scale value, the thicker the layer and the higher the surface concentration.

\subsection{Detection procedure}

The general AIV detection procedure: silicon wafers, used as the substrate, are modified with chemical reagents, resulting in the formation of chemical groups on the surface which can covalently immobilize antibodies against AIV as ligands. The modified wafers are then put in contact with the microfluidic mould so that surfaces of the wafers are patterned to form regular small cells in an array format, the physical size of each cell being about $1.5 \mathrm{~mm} \times 1 \mathrm{~mm}$. To immobilize ligands to form a sensing surface, a solution of antibodies against AIV is pumped into each cell. AIV samples are then added to the sensing surface. After AIV capture, the micro-array is removed from the microfluidic system and examined by IE to determine the results.

\subsubsection{Surface modification}

Silicon wafers were cut into $20 \mathrm{~mm} \times 10 \mathrm{~mm}$ rectangles and rinsed with deionized water. After soaking in piranha solution $\left(30 \% \mathrm{H}_{2} \mathrm{O}_{2}: 98 \% \mathrm{H}_{2} \mathrm{SO}_{4}=1: 3, \mathrm{v} / \mathrm{v}\right.$.) for $30 \mathrm{~min}$ to increase the number of silanol groups on the wafer surface, and rinsing with deionized water and ethanol, the wafers were soaked in a mixture of 3-aminopropyltriethoxy-silane (APTES) and absolute ethanol (APTES:absolute ethanol $=1: 10, v / v$ ) and incubated for $2 \mathrm{~h}$ with gentle agitation. The reaction of APTES with the surface silanol groups resulted in covalent immobilization of $-\mathrm{O}-\mathrm{Si}(\mathrm{OH})_{2}-\left(\mathrm{CH}_{2}\right)_{3}-\mathrm{NH}_{2}$, forming a layer of densely packed amino groups on the surface. After rinsing three times with absolute ethanol, the wafers were incubated in a saturated solution of succinic anhydride in ethanol for at least $3 \mathrm{~h}$ with gentle agitation. The $\mathrm{CH}_{2} \mathrm{CH}_{2} \mathrm{COOCO}-$ group of succinic anhydride reacted with the $-\mathrm{NH}_{2}$ of $-\mathrm{O}-\mathrm{Si}(\mathrm{OH})_{2}-\left(\mathrm{CH}_{2}\right)_{3}-\mathrm{NH}_{2}$ group immobilized on the surface, generating $-\left(\mathrm{CH}_{2}\right)_{3} \mathrm{NH}-\mathrm{CO}\left(\mathrm{CH}_{2}\right)_{2}-\mathrm{COOH}$. Once prepared, wafers were stored in ethanol.

\subsubsection{Ligand immobilization and AIV detection}

When a modified silicon wafer was inserted into a microfluidic system, the carboxyl $\left(-\left(\mathrm{CH}_{2}\right)_{3} \mathrm{NH}-\mathrm{CO}-\left(\mathrm{CH}_{2}\right)_{2}-\mathrm{COOH}\right)$ group on its surface was activated by solution NE. The NE was prepared with N-hydroxysuccinimide (NHS, 0.05 M) and 1-(3dimethylaminopropyl)-3-ethylcarbodiimide hydrochloride (EDC, $0.2 \mathrm{M}$ ) in deionized water, and $10 \mu \mathrm{l}$ of NE solution per unit was then added to the system at a flow rate of $5 \mu \mathrm{l} / \mathrm{min}$ and passed through the surface of the wafer. In the presence of NHS, EDC could transfer carboxyl groups to the Sulfo-NHS ester which could then react with the amino groups of the protein. Subsequently, single chain variable fragment ( $\mathrm{scFv}$ ) molecules and the monoclonal antibody (mAb) 4A4 were immobilized separately as ligands in the micro-array using the microfluidic system. 4A4 reacts specifically with AIV subtype H5N1 in hemagglutination inhibition (HI) assays, as described previously (Wang et al., 2004), while scFv molecules were selected from a synthetic phage display library and were able to recognize a conserved epitope in the influenza A virus hemagglutinin (B. Gao, 
unpublished results). Antibody were diluted in PBST ( $140 \mathrm{mM} \mathrm{NaCl}$, $2.7 \mathrm{mM} \mathrm{KCl}, 10 \mathrm{mM} \mathrm{Na}_{2} \mathrm{HPO}_{4}, 1.8 \mathrm{mM} \mathrm{KH}_{2} \mathrm{PO}_{4}, 1 \%$ Tween 20, $\mathrm{pH}$ 7.3 ) to a concentration of $1 \mathrm{mg} / \mathrm{ml}$, and $10 \mu \mathrm{l}$ per unit scFv was then added at a flow rate of $2 \mu \mathrm{l} / \mathrm{min}$ and immobilized directly on the wafer surface. For oriented immobilization of the $4 \mathrm{~A} 4$, protein $\mathrm{A}(0.25 \mathrm{mg} / \mathrm{ml})$ was added to the corresponding unit $(5 \mu \mathrm{l}$ per unit at $2 \mu \mathrm{l} / \mathrm{min}$ ) and covalently immobilized on the surface. $4 \mathrm{~A} 4$ was then added ( $10 \mu \mathrm{l}$ per unit at $2 \mu \mathrm{l} / \mathrm{min})$ and immobilized through an affinity reaction between the Fc fragment of the 4A4 and protein A (Fig. 1A). Lastly, the surface was blocked by $5 \%(\mathrm{v} / \mathrm{v})$ gelatin $(50 \mu \mathrm{l}$ per unit at $2 \mu \mathrm{l} / \mathrm{min})$. The units were rinsed with PBST ( $20 \mu \mathrm{l}$ per unit at $20 \mu \mathrm{l} / \mathrm{min}$ ) between every two consecutive operation steps. Thus, a multiplex micro-array containing a sensing surface array was formed. The AIV samples were added to the inlet of the microfluidic system. The reference AIV antigens [A/Goose/Guangdong/1/96(H5N1) and A/Chicken/Shanghai/10/01 (H9N2)] were kept separately in our laboratory at a titre of 256 as determined by the hemagglutinin test. Virus particles in solution were captured by the specific ligands when passing over the sensing surface. Subsequently, the wafer was rinsed with deionized water and taken out of the system. After rinsing with plenty of deionized water and drying under nitrogen, the micro-array pattern was detected and recorded as images in gray-scale using IE. Binding of AIV results in a significant increase in the gray-scale value for the appropriate unit of the sensing micro-array.

\section{Results and discussion}

\subsection{Qualitative detection of AIV}

A sensing surface consisting of six units with 4A4 ligands and six units with scFv ligands was prepared (Fig. 1B). Inactivated virus stocks of H5N1 and H9N2 were diluted separately (1:10) with PBST and added to the corresponding sensing unit $(50 \mu l$ per unit at
$2 \mu \mathrm{l} / \mathrm{min})$. The results are shown in Fig. 1B. Compared with controls (a3, b3, c3, d3), samples a1/b1, a2/b2 and c1/d1 had markedly thicker films, with the average gray-scale value showing significant increases, e.g. for row 1 , the mean positive sample (a1/b1) score minus the mean control (a3/b3) score was $31.9\left(P_{2}=0.05\right.$, the subscript of $P$ indicates the quantity of units calculated $P$ value. $P$-value is calculated with Single-factor analysis of variance and $F$ test.), while for row 2 the mean positive sample (a2/b2) score minus the mean control $(\mathrm{a} 3 / \mathrm{b} 3)$ score was $33.2\left(P_{2}=0.038\right)$ and the mean positive sample (c1/d1) score minus the mean control (c3/d3) score for row 3 was $60.2\left(P_{2}=0.002 ; P_{10}=0.000003\right)$. Sample $(\mathrm{c} 2 / \mathrm{d} 2)$ did not show significant increases and increases score were $5.7\left(P_{2}=0.184 ; P_{10}=0.42\right)$. CV (coefficient of variation $) \%=$ SD (standard deviation)/mean $\times 100 \%$. The intraassay CV\% in unit "d1" of detection $\mathrm{H} 5 \mathrm{~N} 1$ is $4.3 \%$ and the interassay CV\% among 10 units of detection $\mathrm{H} 5 \mathrm{~N} 1$ on 5 chips is $12.2 \%$. For the positive response to $\mathrm{H} 5 \mathrm{~N} 1$, the percentage of signal increase in 60.2 /the mean control $(\mathrm{c} 3 / \mathrm{d} 3) \times 100 \%=45.6 \%$ which is much exceed the $\mathrm{CV} \%$, while the negative response of H9N2 is $5.7 /$ the mean control $(\mathrm{c} 3 / \mathrm{d} 3) \times 100 \%=4 \%$ which is less than the CV\%. Thus we can conclude that while the two subtypes (H5 and H9) could be captured by the scFv, they were easily distinguished by the $4 \mathrm{~A} 4$ ligand. Moreover, in the detection of H5N1, the gray-scale value when 4A4 was used as ligand (c1, d1) was markedly higher than that obtained when scFv was used as ligand (a1, b1), also demonstrating that 4A4 was more efficient for H5N1 capture.

Immobilization of ligand can impact its bioactivity and affect the efficiency of AIV detection. Since direct ligand immobilization is simple, we decided to immobilize the scFv and 4A4 directly to the surface. We found that scFv could detect the two subtypes (H5 and H9) effectively, but 4A4 did not detect either subtype. We hypothesize that the functional domain of scFv may be exposed, while that of $4 \mathrm{~A} 4$ is blocked due to steric hindrance and random orientation of the antibody molecules adsorbed on the solid-phase surface (Kanno
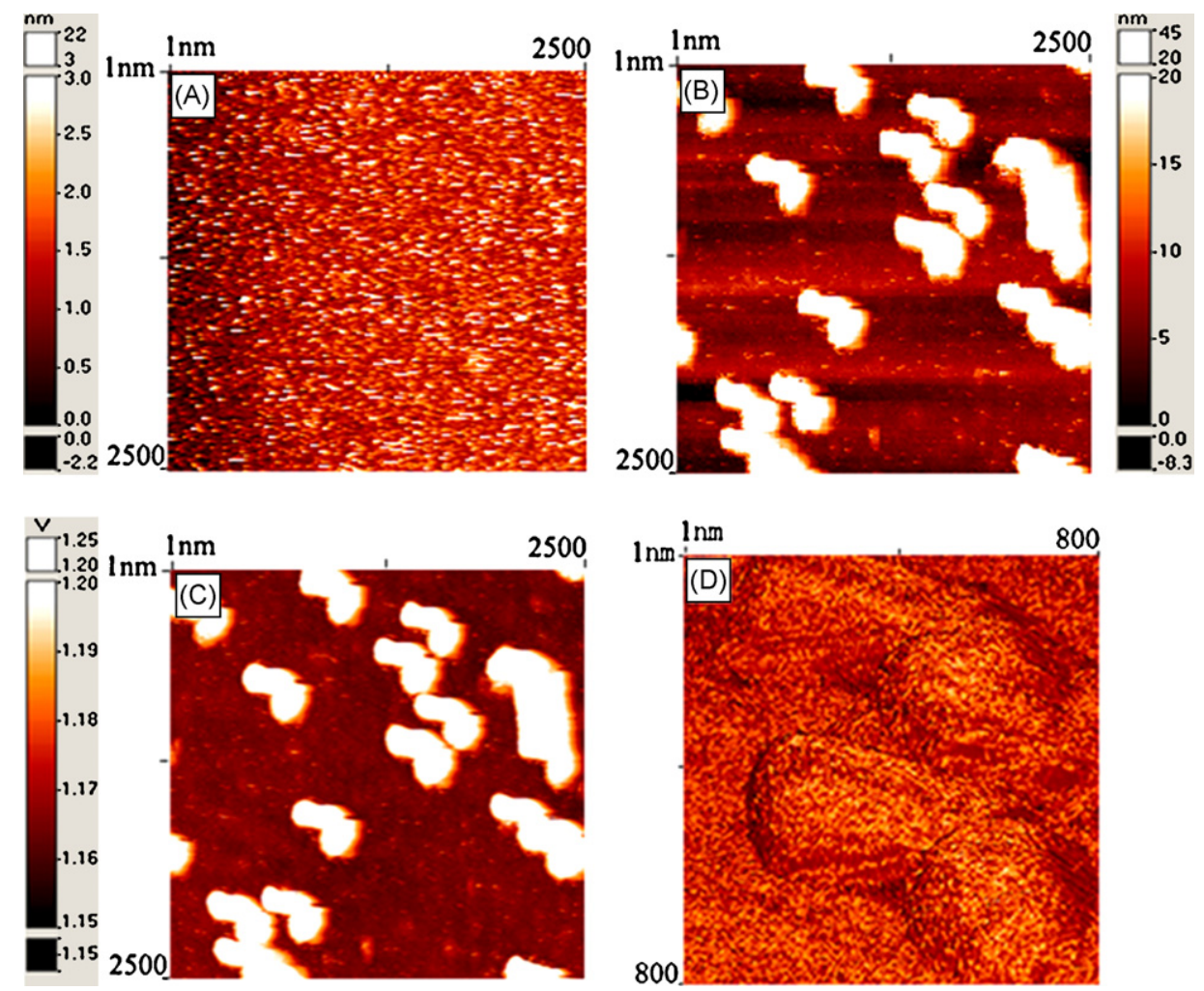

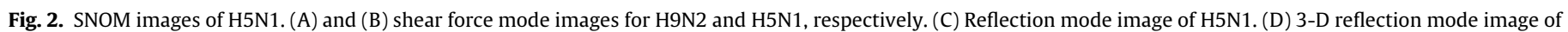
H5N1. 
(A)

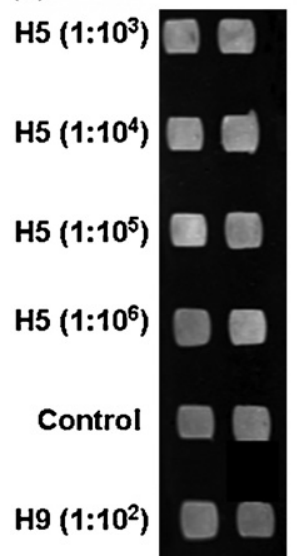

(B)

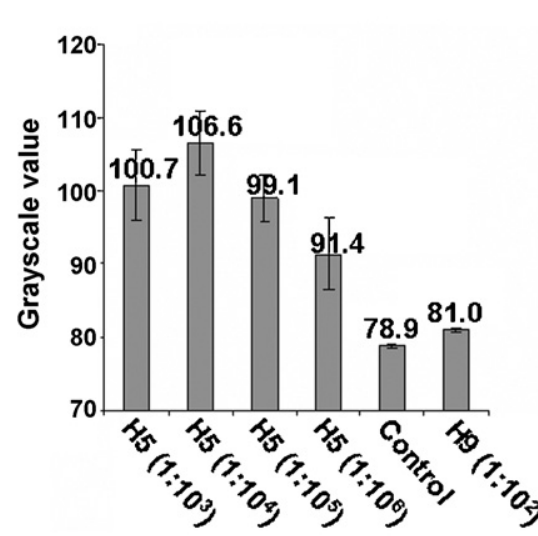

(C)

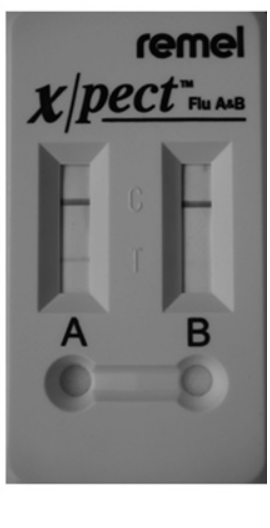

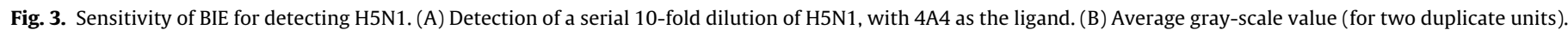

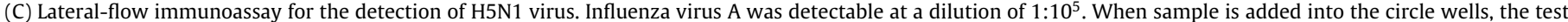

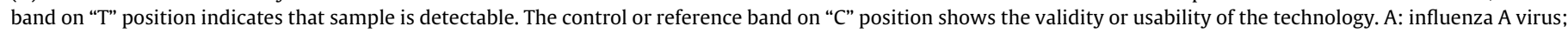
B: influenza B virus.

et al., 2000). Oriented immobilization of antibodies by means of capturing the Fc fragment via an interaction with an adsorbed layer of protein A is an effective way for assaying anti-IgG (Wang and Jin, 2003b). However, scFv does not include an Fc fragment and therefore cannot be immobilized with protein A. 4A4 immobilized via the interaction of protein $A$ and its Fab fragment points away from the substrate, so that its binding with AIV is facilitated, thus allowing successful detection of H5N1. Thus, the oriented immobilization of $\mathrm{mAb} 4 \mathrm{~A} 4$ with protein $\mathrm{A}$ used here is an effective method for assaying $\mathrm{H} 5 \mathrm{~N} 1$.

\subsection{Confirmation of AIV detection by SNOM}

In order to confirm the presence of virus particles on the surface, an H5N1 positive unit (c1, Fig. 1B) was scanned by scanning near-field optical microscopy (SNOM) (Toshiharu and Yoshihito, 2002) using shear force and reflection modes at the same time. Both images are shown in Fig. 2. Large numbers of virus particles of 200-400 $\mathrm{nm}$ in diameter could be seen on the unit in the shear force mode (Fig. 2B), whereas none were present on the negative control unit (Fig. 2A, c2 in Fig. 1B) and no H9N2 particles were captured by the 4A4 layer. A high resolution image was obtained in reflection mode (Fig. 2C and D) which uses direct light beams to "watch" rather than "touch" (shear force mode) the virus and is less disruptive to the bioactivity of biological molecules. The results from both modes were in good agreement, and the size and outline of the virus shown in the micrographs were also consistent with those deduced from electron micrographs of AIV (Nancy, 2007).

\subsection{Sensitivity of BIE}

The sensitivity of BIE for H5N1 detection was compared with that of lateral-flow immunoassay by analyzing detection efficiency for a serial 10-fold dilution of H5N1 inactivated virus stock with $2.56 \times 10^{3} \mathrm{TCID} / \mathrm{ml}$ (Tissue culture infectious dose, TCID). A sensing surface consisting of twelve units with 4A4 ligands was prepared. The ten-fold serial dilution series of the H5N1 inactivated virus stock $\left(1: 10-1: 10^{6}\right.$ diluted in PBST), together with H9N2 (1:10² diluted in PBST), was added to the corresponding sensing units (Fig. 3A). As shown in Fig. 3B, detectable signals were obtained (About 15.9\% increases above the average gray-scale value) even when the dilution was as low as $1: 10^{6}$ (Fig. 3B). The same samples ( $50 \mu \mathrm{l}$ of each) were also detected using a lateral-flow immunoassay (Remel Inc.) with 4A4 following the manufacturer's instructions. Our tests showed that the limit of H5N1 detection in the lateral-flow immunoassay was at a dilution of $1: 10^{5}$ (Fig. 3C). Repeating more than 10 times, our results thus show that the BIE assay was more sensitive in detecting highly pathogenic AIV. On the other hand, Zhang et al. (2005) proposed that if different serial dilutions of samples can be distinguished by BIE, then BIE has the potential to detect unknown concentration of samples, quantitatively.

The micro-arrays developed here have multi-cells immobilized with different ligands for different AIV subtypes. IE can be used to visualize the variation in signal from all units of the microarray with high spatial resolution so that specific binding can be identified. Therefore, different AIV subtypes can simultaneously be identified in one micro-array in less than 10 min from virus hybridization to image capture. So far, our work is just a demonstration for AIV detection with BIE. Unknown or simulated (artificial) samples need to be done in the future for further practical applications.

\section{Conclusion}

We have developed a label-free and multiplex BIE method, using a micro-array and oriented immobilization of $\mathrm{mAb} 4 \mathrm{~A} 4$ via protein A, which provides rapid identification of H5N1 and H9N2 viruses simultaneously. Our results demonstrate that BIE can be used for quantitative detection of more types of virus or virus subtypes using a simple and fast procedure.

\section{Acknowledgements}

The authors acknowledge financial support from the National Basic Research Program of China 2009CB320300, the National High Technology Research and Development Program (863) of China (2006AA02Z4C2), the National Basic Research Program (Project 973) of China (2007CB310505) and the National Key Technologies R\&D Program (2006BAD06A01), Chinese Academy of Sciences (KJCX2-YW-Mo3 and -M04).

\section{References}

Arwin, H., Welinklinstorm, S., Jansson, R., 1993. J. Colloid Interface Sci. 156, 377-382. Bae, Y.M., Oh, B.K., Lee, W., Lee, W.H., Choi, J.W., 2004. Biosens. Bioelectron. 20, 895-902.

Chen, Y.Y., Meng, Y.H., Jin, G., 2007. Appl. Opt. 46, 8475-8481.

Jin, G., 2008. Phys. Status Solidi 205, 810-816. 
Jin, G., Jansson, R., Arwin, H., 1996. Rev. Sci. Instrum. 67, 2930-2936.

Kanno, S., Yanagida, Y., Haruyama, T., Kobatake, E., Aizawa, M., 2000. J. Biotechnol. $76,207-214$.

Lau, L.T., Banks, J., Aherne, R., Brown, I.H., Dillon, N., Collins, R.A., Chan, K.Y., Fung, Y.W., Xing, J., Yu, A.C., 2004. Biochem. Biophys. Res. Commun. 313, 336-342.

Lin, C.H., Hung, C.H., Hsiao, C.Y., Lin, H.C., Ko, F.H., Yang, Y.S., 2009. Biosens Bioelectron. 24, 3019-3024.

Malik Peiris, J.S., 2009. Rev. Sci. Tech. 28 (1), 161-173.

Nancy, T., 2007. http://www.genomenewsnetwork.org/articles/2004/02/20/avian_ flu.php.

Payungporn, S., Chutinimitkul, S., Chaisingh, A., Damrongwantanapokin, S., Buranathai, C., Amonsin, A., Theamboonlers, A., Poovorawan, Y., 2006. J. Virol. Methods 131, 143-147.

Qi, C., Duan, J.Z., Wang, Z.H., Chen, Y.Y., Zhang, P.H., Zhan, L., Yan, X.Y., Cao, W.C., Jin, G., 2006. Biomed. Microdevices 8, 247-253.
Qi, C., Zhu, W., Niu, Y., Zhang, H.G., Zhu, G.Y., Meng, Y.H., Chen, S., Jin, G., 2009a. J. Viral Hepatitis 16, 822-832.

Qi, C., Lin, Y., Feng, J., Wang, Z.H., Zhu, C.F., Meng, Y.H., Yan, X.Y., Wan, L.J., Jin, G., 2009b. Virus Res. 140, 79-84.

Stenberg, M., Nygren, H., 1983. J. dé Physique C10, 83-86.

Toshiharu, S., Yoshihito, N., 2002. JSAP International 5.

Wang, C.B., Tian, X.S., Cao, Z., Yu, X.L., Sun, M., Chen, X.Z., Wang, H.W., Tian, K.G., 2004. Acta Lab. Anim. Sci. Sinica 12, 204-207.

Wang, Z.H., Jin, G., 2003a. Anal. Chem. 75, 6119-6123.

Wang, Z.H., Jin, G., 2003b. J. Biochem. Biophys. Methods 57, 203-211.

Wang, Z.H., Meng, Y.H., Ying, P.Q., Qi, C., Jin, G., 2006. Electrophoresis 27, 40784085.

World Health Organization, 2009. http://www.who.int/csr/disease/avian_influenza/ country/cases_table_2009_09_24/en/index.html.

Zhang, H.G., Qi, C., Wang, Z.H., Jin, G., Xiu, R.J., 2005. Clin. Chem. 51, 1038-1040. 